\title{
Algumas características da reprodução e ontogênese de Epilachna paenulata (Germar) (Coleoptera, Coccinellidae, Epilachninae) ${ }^{1}$
}

\author{
Norma G. Ganho 2,3 \\ Renato C. Marinoni ${ }^{2,4}$
}

\begin{abstract}
Some reproductive and ontogenetic characteristics of Epilachna paenulata (Germar, 1824) (Coleoptera, Coccinellidae, Epilachninae). Epilachna paenulata (Germar, 1824) was reared feeding on pumpkin leaves (Cucurbita pepo Lourt.) in laboratory conditions at temperatures of $20 \pm 0,5^{\circ} \mathrm{C}$, relative humidity of $65 \pm 0,5 \%$ and 12 hour photoperiod. Groups of fecunded and non-fecunded females were studied. The ovarioles showed four developing stages during egg production, morphologically alike for fecunded and non-fecunded females, but differing in the duration time of the stages. Each ovarium has 24 ovarioles on average, less than any other species known into the genus, and all ovarioles are physiologically active at each oviposition. The mean number of ovarioles is lower when compared with the other species of Epilachninae known. Fecunded and non-fecunded females showed a similar preoviposition period, but longer than any other species known into the genus. The interoviposition period is shorter among fecunded females than non-fecunded and among other species of the genus. The number of ovipositions and eggs per oviposition and the daily oviposition rate are higher among fecunded females than non-fecunded and any other species of the genus. Differing from other Coccinellidae species, the mean eggs width do not change between the first and second ovipositions. The eggs handling decrease the larvae viability. The differences observed in the number of eggs per oviposition, in the interoviposition periods and the daily oviposition rate between fecunded and non-fecunded females showed that mate changes the female reprodutive capacity.
\end{abstract}

KEY WORDS. Epilachninae, reproduction, ovariole development, mate influence, viability

A subfamília Epilachninae reúne espécies fitófagas, alimentando-se na maioria das vezes de folhas de cucurbitáceas e solanáceas (HODEK \& HONEK 1996). A tribo Epilachnini tem maior ocorrência na Região Andina, principalmente no centro sul do Peru, tudo indicando ser este o centro de especiação do grupo; observa-se também uma relação entre as distribuições geográficas de cucurbitáceas e solanáceas (também originárias da mesma região) e a destes insetos (GORDON 1976). Algumas espécies, mais importantes sob o ponto de vista agronômico, têm seus aspectos biológicos mais estudados, como: Epilachna varivestis Mulsant, 1850 , que se alimenta das folhas de muitas variedades de feijoeiros e ocorre na

1) Contribuição número 1195 do Departamento de Zoologia, Universidade Federal do Paraná.

2) Departamento de Zoologia, Universidade Federal do Paraná. Caixa Postal 19020, 81531-990 Curitiba, Paraná, Brasil.

3) Curso de Pós-graduação em Ciências Biológicas, Entomologia. Universidade Federal do Paraná. Bolsista da CAPES.

4) Bolsista do CNPq. 
Região Neártica; Epilachna vigintioctopunctata Fabricius, 1775, que ocorre na Região Oriental e come folhas de plantas de batata-inglesa e beringela (HOWARD 1936; AUCLAIR 1959; KATAIAMA et al. 1979; JONES et al. 1981; WiLSON et al. 1982; NAKAMURA 1983; NAKAMURA et al. 1988; RAJAGOPAL \& TRIVEDI 1989). Na Região Neotropical, as espécies mais estudadas foram E. paenulata (Germar, 1824), que se alimenta de folhas de aboboreira (Cucurbita pepo Linnaeus, C. maxima Duchesne, C. moscata Duchesne, Lagenaria vulgaris Ser.), de chuchuzeiro (Sechium edule (Jacq.) Sw.), de pepineiro (Cucumis sativus L.); Epilachna cacica (Guérin, 1844) que se alimenta de folhas de Cucurbita pepo e C. moschata; Epilachna spreta Mulsant, 1850, que tem como alimento folhas de Cucurbita pepo; E. clandestina Mulsant, 1850, folhas de Cayaponia ficifolia (Chambers) Cogn. e Cucurbita pepo (FONSECA \& AUTUORI 1931; PRECETTI et al. 1977; Silva et al. 1968; GORDON 1976; ALMEIDA \& MARINONI 1986; ALMEIDA \& RIBEIRO 1986; MARINONI \& RIBEIRO 1987; RIBEIRO \& ALMEIDA 1989; MARINONI \& GIAMBARRESI 1992). Os estudos anteriores sobre a biologia de Epilachna paenulata trataram das condições de desenvolvimento da espécie (tamanho, tempo de desenvolvimento das fases larvais) em diferentes condições de temperatura (ALMEIDA \& MARINONI 1986); e da viabilidade da espécie em quatro diferentes plantas hospedeiras (MARINONI \& RIBEIRO 1987). O presente trabalho tem como objetivo aumentar os conhecimentos sobre o comportamento reprodutivo desta espécie através de estudos do desenvolvimento ovariano, oviposição, viabilidade e longevidade.

\section{MATERIAL E MÉTODOS}

Adultos, pupas, larvas e ovos de Epilachna paenulata foram coletados em folhas de aboboreira (Cucurbita pepo), nas regiões urbanas de Curitiba e de São José dos Pinhais, Paraná. Logo após a coleta os exemplares foram acondicionados em recipientes de plástico semitransparentes de $500 \mathrm{ml}$, com tampa perfurada. Em laboratório do Departamento de Zoologia da Universidade Federal do Paraná, o material coletado e todos os demais experimentos foram mantidos em câmara com temperatura constante de $20 \pm 0,5^{\circ} \mathrm{C}$, fotoperíodo de $12 / 12$ horas e umidade relativa de $65 \pm 5 \%$. Adultos e larvas foram alimentados diariamente com folhas de aboboreira, nas mesmas condições descritas por ALMEIDA \& MARINONI (1986) e MARINONI \& RIBEIRO (1987).

Os exemplares utilizados nos experimentos foram obtidos a partir das oviposições das fêmeas coletadas no campo, colocadas em placa de Petri com papel filtro umedecido até a eclosão das larvas. Estas foram transferidas para frascos plásticos com folhas de aboboreira, que eram trocadas diariamente. Este procedimento foi repetido até que as larvas empupassem. Cada pupa foi isolada em placa de Petri forrada com papel filtro umedecido, para se registrar a emergência do adulto, e evitar a cópula.

Os estudos desdobraram-se em quatro experimentos, com diferentes objetivos primários e diferentes condições de manejo, visando obter informações sobre número de posturas; período de pré-oviposição; intervalo entre as posturas; longevidade e número total de ovos das fêmeas, com a identificação das fases do desenvolvimento ovariano em fêmeas fecundadas e não fecundadas; viabilidade dos ovos, relação entre a largura dos ovos e o tamanho das larvas de primeiro ínstar. 
Primeiro experimento - Objetivo primário: verificar a viabilidade de ovos não manuseados. Iniciado com oito casais, um em cada pote; feita a postura esta era identificada por um número de ordem (número de posturas) e mantida em placa de Petri com papel filtro umedecido até a eclosão das larvas que eram contadas. Registravam-se ainda: período de pré-oviposição; intervalo entre as posturas; número total de ovos por fêmea; e longevidade.

Segundo experimento - Objetivo primário: medir a largura do ovos. Iniciado com dez casais, um em cada pote. Os ovos foram medidos através de ocular micrométrica acoplada a um microscópio estereoscópico. Como os ovos são postos acolados foi necessária a separação com o uso de agulha e pincel. Foram feitos os demais registros como no primeiro experimento.

Terceiro experimento - Objetivo primário: medir a largura dos ovos e a distância entre os alvéolos antenais da cápsula cefálica das larvas de primeiro ínstar. Iniciado com dez casais, um em cada pote. Apenas os ovos em condições de fácil visualização, principalmente os não acolados, foram medidos, como no segundo experimento. Foram feitos os demais registros como no primeiro experimento.

Quarto experimento - Objetivo primário: identificação das diferentes fases do desenvolvimento ovariano. Foram estabelecidos dois grupos para estudo, um deles com casais para que houvesse fecundação dos ovos e o outro com fêmeas virgens. Nos dois grupos, as fêmeas foram dissecadas para a identificação dos diferentes estágios de desenvolvimento dos ovaríolos, em intervalos determinados pelo número de seqüência da postura. Foram observados os estágios dos ovaríolos em dez fêmeas fecundadas e em oito fềmeas não fecundadas. Foram registrados os dados referentes ao número total de ovos por fềmea e longevidade de quatro fêmeas não fecundadas.

As dissecações para estudo dos ovários e determinação do estágio de desenvolvimento foram feitas em placa de Petri, contendo fundo de parafina e água destilada. Foi feito um corte longitudinal dos tergos abdominais, no sentido póstero-anterior; foram destacados os tergos e removidas as estruturas que se sobrepõem ao sistema reprodutor; e rompidos os ligamentos que unem os filamentos terminais dos ovaríolos ao tórax. Após a retirada do sistema reprodutor, cortado na base da vagina, o mesmo foi corado por alguns segundos com "sudan black", para melhor visualização da membrana que envolve os ovaríolos e do contorno dos ovos.

Nas discussões, quando não houver referência expressa ao autor, as citações a espécies de Epilachna devem ser creditadas aos seguintes autores: Epilachna varivestis Mulsant, 1850 a AUCLAIR (1959); Epilachna cacica (Guérin, 1844) a PRECETTI et al. (1977); Epilachna chrysomelina Fabricius, 1775 a ALI \& EL SAEADY (1981); Epilachna clandestina Mulsant, 1850 a MARINONI \& GIAMBARRESI (1992).

\section{RESULTADOS E DISCUSSÕES}

\section{Estágios de desenvolvimento dos ovários}

Fêmeas fecundadas e não fecundadas foram periodicamente dissecadas para a identificação das diferentes fases de desenvolvimento dos ovários. Em fêmeas recém-emergidas, os ovários apresentam-se envoltos por traquéias e os ovaríolos mostram-se indiferenciados e translúcidos. Estas condições, segundo PHOOFOLO et al. (1995), também são observadas nas fêmeas em diapausa. 
Para a caracterização dos estágios dos ovaríolos foi criada uma classificação, baseada em parte naquela proposta por PHOOFOLO et al. (1995), pela utilização da variação da forma e da cor dos folículos, desde o seu surgimento até o amadurecimento.

Os ovaríolos de E. paenulata apresentam quatro estágios de desenvolvimento (Fig. 1). Estágio I: cada ovariolo apresenta apenas um folículo, o primário (início da diferenciação: esférico e translúcido); observado no período de pré-oviposição. Estágio II: cada ovaríolo contém dois folículos no vitelário; o folículo secundário (início da vitelogênese: oval e bege), mais proximal, seguido pelo primário, esférico; no folículo secundário, com o desenvolver da vitelogênese, aparece a coloração amarela e a consistência de gel. Estágio III: três folículos no vitelário; o folículo terciário (vitelogênese quase completa: elipsoidal e amarelo) maior que os anteriores e mais amarelo. Estágio IV: três folículos no vitelário; o folículo terciário já transformado em quaternário (vitelogênese completa e corionização: elipsoidal e amarelo) é maior que os outros dois (primário e secundário) e a cor é mais fortemente amarelada. A este estágio segue-se a ovulação e oviposição.

As fases de desenvolvimento dos ovaríolos mostraram-se morfologicamente semelhantes entre fêmeas fecundadas e não fecundadas, porém com diferenças no tempo de duração de cada estágio. O trabalho de PHOOFOLO et al. (1995) mostra que a espécie Coccinella septempunctata Linnaeus, 1758, apresenta também quatro estágios de desenvolvimento dos ovaríolos.

No presente estudo, foi observada uma média de 24 (19-32) ovaríolos por ovário. ROBERTSON (1961) se refere a uma média de 27 ovaríolos por ovário em espécies de Epilachninae, diferente de Coccinellinae com uma média de 16 ovaríolos. DOBZHANSKI (segundo ROBERTSON 1961) encontrou de 30 a 33 ovaríolos em Epilachna pusillanima Mulsant, 1850; 27 a $28 \mathrm{em}$ Epilachna vigintioctomaculata Motschulsky, 1857; e 41 a 43 em Epilachna undecimvariolata Boisduval, 1835. Exceto por esta última espécie, o número médio de ovaríolos é relativamente semelhante dentro da subfamília Epilachninae. RHAMHALINGHAN (segundo HodEK \& HONEK 1996), afirmou que em Coccinella septempunctata o número de ovaríolos varia sazonalmente, de 26 a 82 ovariolos por ovário; esta variação é resultado de uma alteração no comportamento reprodutivo com a diminuição de alimento. Apesar do número de ovaríolos observados em E. paenulata indicar uma amplitude de variação superior àquelas observadas nas espécies de Epilachna acima, não é possível indicar o alimento como uma possível causa, pois este foi fornecido regularmente e de forma semelhante a todas as fêmeas.

STEWART et al. (1991) estudaram onze espécies de coccinelídeos afidófagos e observaram que o número de ovos por postura é aproximadamente metade do número de ovaríolos, enquanto a outra metade prepara-se para nova oviposição. Em fêmeas fecundadas de $E$. paenulata todos os ovaríolos são fisiológicamente ativos a cada postura. Vários folículos desenvolvem-se simultaneamente em diferentes estágios em cada ovaríolo, permitindo uma contínua produção de ovos.

\section{Períodos reprodutivos (Tab. I)}

$\mathrm{O}$ período médio de pré-oviposição (emergência do imago à primeira ovi-

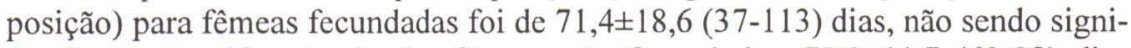

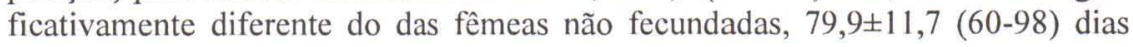




\section{A Estágio de ovaríolo B}
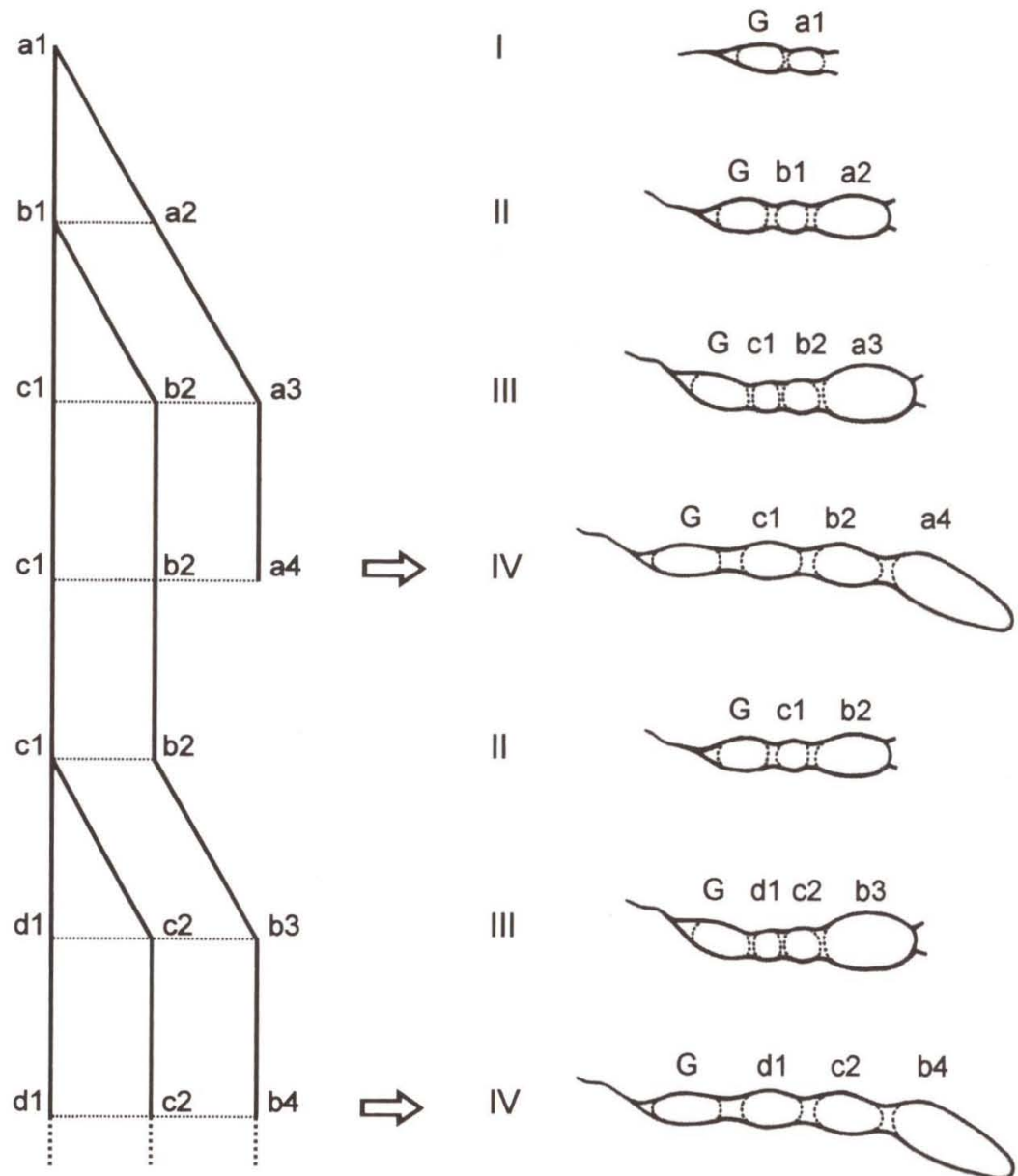

III

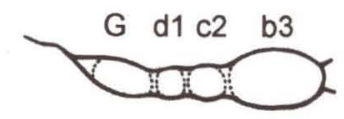

Fig. 1. Epilachna paenulata. (A) Fluxograma dos estágios de cada foliculo ovariano; (B) folículos ovarianos em estágios de desenvolvimento correspondente ao fluxograma. (G) Germário; (letras minúsculas) folículo; (algarismo arábico) fase do folículo; (algarismo romano) estágio do ovaríolo; (seta) postura do ovo.

(Teste $\mathrm{F}, \mathrm{p}=0,24$; teste $\mathrm{T}, \mathrm{p}=0,25$ ). Estes períodos são superiores aos observados em E. chrysomelina (12,2 $\pm 0,6)$; em E. varivestis (50-52 dias); e em E. clandestina (53-62 dias).

O estudo da fisiologia dos ovaríolos mostrou que estes ao atingirem o estágio IV, eliminam o folículo quaternário (ovo), e retornam ao estágio II, com dois folículos; a seguir, o folículo secundário se desenvolve em folículo terciário e o folículo primário em secundário; e surge um novo folículo (primário) (Fig. 1). O tempo que os ovaríolos levam para novamente atingir o estágio IV, equivalente ao 
intervalo entre as posturas, foi em média de 5,6 6 2,1 (3,1-14,0) dias entre as fềmeas fecundadas, e significativamente menor que entre as não fecundadas, $15,1 \pm 5,0$ $(8,0-23,0)$ dias (Teste $F, p<0,001$; teste $t, p=0,002)$. Os valores observados para as fêmeas fecundadas são menores que os de $E$. cacica, 6,5 51,0 dias, e os de $E$. clandestina, de 11,9 (5,1-19,5) dias.

O período total de oviposição entre as fêmeas fecundadas foi de $47,6 \pm 18,94$ dias; entre as fêmeas não fecundadas houve um aumento do período, 72,5 $\pm 51,4$ dias.

Tabela I. Epilachna paenulata. Dados de pré-oviposição, intervalos entre as posturas, período de oviposição, número de posturas por fêmea, número de ovos por postura, número de ovos postos por dia, número total de ovos por fêmea, longevidade, largura dos ovos das $1^{\mathrm{a}}$ e $2^{\mathrm{a}}$ posturas, largura da fronte do primeiro instar larval. (F) Fêmeas fecundadas; (NF) fêmeas não fecundadas.

\begin{tabular}{|c|c|c|c|c|c|c|}
\hline & & $\mathrm{N}$ & Média & Desvio padrão & Amplitude & Teste $\mathrm{t}$ \\
\hline \multirow[t]{2}{*}{ Pré-oviposição (dias) } & $\mathrm{F}$ & 44 & 71,40 & 18,60 & $37,00-113,00$ & \\
\hline & NF & 7 & 79,90 & 11,70 & $60,00-98,00$ & $p=0,250$ \\
\hline \multirow[t]{2}{*}{ Intervalo entre as posturas (dias) } & $\mathrm{F}$ & 42 & 5,60 & 2,10 & $3,10-$ & \\
\hline & NF & 7 & 15,10 & 5,00 & $8,00-$ & $p=0,002$ \\
\hline \multirow[t]{2}{*}{ Periodo de oviposiçăo (dias) } & $\mathrm{F}$ & 20 & 47,60 & 18,90 & $19,00-81,00$ & \\
\hline & NF & 4 & 72,50 & 51,40 & $18,00-135,00$ & \\
\hline \multirow[t]{2}{*}{ Número de posturas por fêmea } & $\mathrm{F}$ & 33 & 12,50 & 4,40 & $3,00-20,00$ & \\
\hline & NF & 4 & 5,80 & 3,40 & $3,00-$ & \\
\hline \multirow[t]{2}{*}{ Número de ovos por postura } & $\mathrm{F}$ & 33 & 53,90 & 4,90 & $39,80-$ & \\
\hline & NF & 7 & 38,90 & 9,50 & $23,00-$ & $p=0,005$ \\
\hline \multirow[t]{2}{*}{ Número de ovos postos por dia } & $\mathrm{F}$ & 20 & 12,00 & 3,60 & $6,50-$ & \\
\hline & NF & 7 & 3,50 & 1,50 & $1,50-\quad 5,40$ & $p<0,001$ \\
\hline \multirow[t]{2}{*}{ Número total de ovos por fêmea } & $\mathrm{F}$ & 20 & 555,00 & 234,90 & $175,00-1108,00$ & \\
\hline & NF & 4 & 196,00 & 100,40 & $89,00-319,00$ & \\
\hline \multirow[t]{2}{*}{ Longevidade (dias) } & $\mathrm{F}$ & 20 & 138,40 & 34,00 & $94,00-219,00$ & \\
\hline & NF & 4 & 203,00 & 58,20 & $146,00-281,00$ & \\
\hline \multirow[t]{3}{*}{ Largura dos ovos da primeira e segunda posturas } & Primeira & 20 & 0,69 & 0,02 & $0,64-\quad 0,72$ & \\
\hline & Segunda & 20 & 0,68 & 0,04 & $0,61-$ & $p=0,407$ \\
\hline & & 40 & 0,68 & 0,03 & $0,61-$ & \\
\hline Largura da fronte do primeiro instar larval & & 10 & 0,34 & 0,01 & $0,32-$ & \\
\hline
\end{tabular}

\section{Número de posturas e de ovos (Tab. I)}

O número de posturas das fêmeas fecundadas foi superior, quase duas vezes maior, que o das fềmeas não fecundadas $(12,5 \pm 4,4: 5,8 \pm 3,4)$; e maior que o observado em $E$. clandestina, que foi de 8,0 posturas por fêmea.

O número médio de ovos por postura por fêmea foi de $53,8 \pm 4,9(39,8-62,4)$ dentre as fêmeas fecundadas e significativamente superior ao observado entre as não fecundadas, 38,9 9 9,5 (23,0-48,7) (Teste $F, p=0,014$; teste $t, p=0,005$ ). Epilachna clandestina apresentou 28,1 (20,1-33,0) ovos por postura por fêmea; e em $E$. varivestis houve uma variação de 40 a 60 ovos.

A relação entre o período de oviposição e o número total de ovos postos entre as fềmeas fecundadas foi de $12,0 \pm 3,6(6,5-18,6)$ ovos por dia por fêmea, significativamente superior ao das fêmeas não fecundadas, 3,5 $\pm 1,5(1,5-5,4)$ (Teste $F, p=$ $0,04$; teste t, $\mathrm{p}<0,001)$. Em E. clandestina foi observada uma média de 2,8 $(1,4-5,7)$ ovos por dia por fêmea. 
O número total de ovos por fềmea fecundada foi de 555 $\pm 234,9$ (175-1108) ovos; e entre as fềmeas não fecundadas foi cerca de três vezes menor, $196 \pm 100,4$ (89-319) ovos por fềmea. O total de ovos das fêmeas fecundadas foi 2,5 vezes maior do que o observado para $E$. clandestina (216: 54-406); e 1,8 vezes maior que o de E. chrysomelina que foi de $308,3 \pm 22,6$.

\section{Influência da cópula na reprodução}

Os valores significativamente diferentes do intervalo entre as posturas, do número de ovos por postura, e do número de ovos postos por dia, observados entre as fêmeas fecundadas e não fecundadas de E. paenulata, indicam que a cópula altera a capacidade reprodutiva da fêmea. As fêmeas fecundadas ovipuseram mais, com uma menor variação no número de ovos por postura, estando de acordo com o observado em Tribolium confusum Duval, 1868 (Coleoptera, Tenebrionidae), em qúe com a cópula houve aumento de produção de ovos de cerca de 20 vezes (PARK 1934). Estas observações foram confirmadas por SCHNEIDER (1941). Múltiplas cópulas em Acanthocelides obtectus (Say, 1831) (Coleoptera, Bruchidae) resultaram num aumento da sobrevivência da fêmea, na produção e no tamanho dos ovos, além da sobrevivência das larvas (HUIGNARD 1983). A influência da cópula também foi descrita em Orthoptera (FrIEDEL \& CEDRIC 1977; SIMMONS 1988); em Diptera (LEOPOLD 1976; KLOWDEN \& CHAMBERS 1991); e ainda em Blattodea e Lepidoptera (WHEELER 1996). Esta influência pode ser devida à passagem de substâncias químicas que, como demostrado por HuIGNARD (1983), em Bruchidae, são transferidas pelo espermatóforo para a hemolinfa da fêmea, resultando num incremento da atividade ovariana, sendo responsáveis pela estimulação da oogênese, com alguns elementos sendo incorporados aos oócitos. WHEELER (1996), em estudo revisivo da literatura sobre a nutrição na oogênese, destaca que a contribuição nutricional que o macho transfere durante a cópula, freqüentemente tem um impacto na fecundidade da fềmea que está sob condições de "stress" de nutrientes.

O aumento no intervalo entre as posturas e a diminuição do número de ovos por postura, observados em E. paenulata, evidencia uma possível estratégia de preservação de oócitos até que ocorra a cópula.

\section{Largura dos ovos e da fronte da larva (Tab. I)}

A largura média dos ovos de E. paenulata foi de $0,68 \pm 0,03 \mathrm{~mm}(0,61-0,76)$, variando dentro de uma mesma postura; e os ovos da primeira e segunda posturas não apresentaram larguras significativamente diferentes (Teste $\mathrm{F}, \mathrm{p}=0,089$; teste $\mathrm{t}, \mathrm{p}=0,407)$. A largura dos ovos anotada para $E$. clandestina foi um pouco maior, $0,69 \mathrm{~mm}(0,64-0,75)$. A inexistência de diferença de larguras dos ovos entre as posturas não acompanha o observado por MARINONI \& GIAMBARRESI (1992) que constataram haver uma maior largura média em ovos da primeira postura em relação às segunda ou terceira posturas de E. clandestina; e por STEWART et al. (1991) em quatro espécies de coccinelídeos, Adalia bipunctata (Linnaeus 1758); Propylea quatuordecimpunctata (Linnaeus 1758); Adalia decimpunctata (Linnaeus 1758) e Coccinella trifasciata (Linnaeus 1758), em que na primeira postura os ovos foram um pouco menores que o restante das posturas. 
A distância inter-alveolar da cápsula cefálica (largura da fronte) de larvas de primeiro instar de $E$. paenulata, provenientes de ovos mensurados, foi de $0,34 \pm 0,007 \mathrm{~mm}(0,32-0,34)$. ALMEIDA \& MARINONI (1986), para esta mesma espécie, nas mesmas condições dos experimentos deste trabalho, encontraram um valor médio um pouco superior, $0,38 \mathrm{~mm}$; para as espécies $E$. spreta e E. cacica, os valores foram ainda maiores, $0,46 \mathrm{~mm}$ e $0,48 \mathrm{~mm}$, respectivamente. A outra espécie com largura de fronte conhecida, E. clandestina, alcança $0,42 \mathrm{~mm}$. Estes valores confirmam E. paenulata como a espécie de menor tamanho dentre as já estudadas. O coeficiente de correlação linear entre a largura média da fronte das cápsulas cefálicas de larvas de primeiro instar e a largura média dos ovos dos quais provieram indicou não haver correlação entre as mesmas $(r=0,245)$.

\section{Viabilidade dos ovos}

A viabilidade dos ovos não manuseados foi significativamente superior à dos ovos que foram manuseados para mensuração (5987 ovos - 2897 larvas; 6588 ovos - 1513 larvas) (Teste $X^{2}, p<0,001$ ), numa relação de $49 \%$ para $23 \%$. Além do manuseio do ovo pelo toque de instrumental para ajustar a posição para mensuração, o fator que possivelmente mais influenciou negativamente a viabilidade foi a incidência de luz de lâmpada de filamento incandescente, que aquece o ovo por mais rápido que seja o procedimento; a permanência sob a luz da lâmpada leva à murchidão do ovo.

O valor obtido para ovos não manuseados de E. paenulata foi próximo ao encontrado para E. clandestina, $53 \%$, considerando apenas as posturas viáveis; e muito superior ao de E. cacica, $8,7 \%$.

Estabelecendo-se a comparação entre valores do período de oviposição; do número de ovos por postura; e da longevidade entre as espécies $E$. paenulata e $E$. clandestina, observa-se que: o período médio de oviposição de $E$. paenulata é menor (47,5 x 74,6 dias); o número de ovos postos em E. paenulata é maior $(53,9 \times 28,1$ ovos); e a longevidade de E. paenulata é menor (138 x 196 dias). Estes dados estão de acordo com as observações de CROWSON (1981) de que espécies de Coleoptera com vida curta põe ovos em grande número, e que fêmeas com menor número de ovos por postura têm um período relativamente longo de vida e de produção de ovos.

\section{Longevidade}

A longevidade observada entre as fêmeas fecundadas foi de $138 \pm 33,1$ (94-219) dias, e entre as não fecundadas foi de 203 $\pm 50,4$ (146-281) dias. Os dias de longevidade observados para E. clandestina variou entre 146 e 341 dias.

\section{CONCLUSÕES}

A espécie Epilachna paenulata (Germar, 1824) apresentou as seguintes características de reprodução e ontogênese: A) os ovaríolos têm quatro estágios de desenvolvimento e são semelhantes entre as fêmeas fecundadas e não fecundadas, com diferenças apenas no tempo de duração dos estágios; B) todos os ovaríolos são fisiológicamente ativos a cada postura em fềmeas fecundadas; $\mathrm{C}$ ) o número médio de ovariolos é inferior à média dentro da subfamília Epilachninae; D) o período de 
pré-oviposição é semelhante entre as fêmeas fecundadas e não fecundadas, e superior ao de outras espécies do gênero conhecidas; E) o intervalo entre as posturas é menor entre as fêmeas fecundadas, e menor que o intervalo de outras espécies do gênero conhecidas; F) o número de posturas, o número de ovos por postura e o número de ovos postos por dia por fêmea fecundada são superiores aos da fềmea não fecundada, e ao de outras espécies do gênero conhecidas; G) a largura média dos ovos de E. paenulata não varia entre as primeira e segunda posturas diferentemente de outras espécies de Coccinellidae; $H$ ) não há correspondência entre a largura do ovo e o tamanho da larva gerada; I) o manuseio dos ovos implicou na redução da viabilidade da emergência de larvas.

Os dados referentes ao número de ovos por postura, intervalo entre as posturas, e número médio de ovos postos por dia entre fêmeas fecundadas e não fecundadas indicam que a cópula altera a capacidade reprodutiva da fêmea.

O comportamento reprodutivo de Epilachna paenulata é semelhante ao de outras espécies de Coleoptera que tendo muitos ovaríolos põem ovos em grande número, e apresentam vida curta, enquanto as que põem poucos ovos normalmente têm um período relativamente longo de produção de ovos e vida longa.

\section{REFERÊNCIAS BIBLIOGRÁFICAS}

ALI, M.A. \& A.A. EL-SAEADY. 1981. Influence of temperature, photoperiod and host-plant on the bionomics of the melon ladybird Epilachna chrysomelina (F.) (Coleoptera: Coccinellidae). Z. Angew. Entomol. 91: 256-262.

AlmEIDA, L.M. \& C.S. Ribeiro. 1986. Morfologia dos estágios imaturos de Epilachna cacica Guérin, 1844 (Coleoptera: Coccinellidae). Revta bras. Ent. 30 (1): 43-49.

Almeida, L.M. \& R.C. MARINONI. 1986. Desenvolvimento de três espécies de Epilachna (Coleoptera: Coccinellidae) em três combinações de temperatura e fotoperíodo. Pesq. Agropec. Bras. 21 (9): 927-939.

AUCLAIR, J.L. 1959. Life-history, effects of temperature and relative humidity, and distributionof the mexican bean beetle Epilachna varivestis Mulsant (Coleoptera: Coccinellidae) in Quebec, with a review of the pertinent literature in North America. Ann. Entomol. Soc. Que. 5: 18-43.

Crowson, R.A. 1981. The Biology of the Coleoptera. London, Academic Press, XII+802p.

FonSECA, J.P. \& M. AUTUORI. 1931. Contribuição para a biologia de Solanophila clandestina (Mulsant) (Coccinellidae: Coleoptera). Rev. Entomol., Rio de Janeiro, 1 (2): 219-224.

Friedel, T. \& G. CEDRIC. 1977. Contribution of male-produced proteins to vitellogenesis in Melanoplus sanguinipes. Jour. Insect Physiol. 23: 145-151.

Gordon, R.D. 1976. A revision of the Epilachninae of the western hemisphere (Coleoptera: Coccinellidae). Tech. Bull. 1493, ARS, USDA, Washington.

Hodek, I. \& A. HONEK. 1996. Ecology of Coccinellidae. London, Kluwer Academic Publishers, XVI+464p.

Howard, N.F. 1936. Feeding of the Mexican bean betle larva. U.S. Dep. Agric. Farmers' Bull. 1624: 1-3.

Huignard, J. 1983. Transfer and Fate of Male Secretions Deposited in Spermatophore of Females of Acanthoscelides obtectus Say (Coleoptera: Bruchidae). Jour. Insect. Physiol. 29 (1): 55-63.

JonES, G.C.; M.P. Hoggard \& M.S. Blum. 1981. Pattern and Process in Insect Feeding Behaviour Bean Beetle, Epilachna varivestis. Entomol. Exp. \& Appl. 30: 254-264.

KATAIAMA, K.; R.E. STINNER \& R.L. RABB. 1979. Effects of temperature, humidity and soybean maturity on longevity and fecundity of the adult Mexican bean beetle, Epilachna varivestis Mulsant. Environ. Entomol. 8: 458-464. 
Klowden, M.J. \& G.M. Chambers. 1991. Male accessory gland substances activate egg development in nutritionally stressed Aedes aegypti mosquitoes. Jour. Insect Physiol. 37 (10): 721-726.

LEOPOLD, R.A. 1976. The role of male accessory glands insect reproduction. Annu. Rev. Entomol. 21: 199-221.

Marinoni, R.C. \& C.S. Ribeiro. 1987. Aspectos bionômicos de Epilachna paenulata (Germar, 1824) (Coleoptera: Coccinellidae) em quatro diferentes plantas-hospedeiras (Cucurbitaceae). Revta bras. Ent. 31 (3): 421-430.

Marinoni, R.C. \& N. Glambarresi. 1992. Sobre a oviposição e ontogenia de Epilachna clandestina (Mulsant, 1850) (Coleoptera: Coccinellidae). Revta bras. Ent. 36 (3): 535-540.

NAKAMURA, K. 1983. Comparative studies on population dynamics of closely related phytophagous lady beetles in Japan. Res. Popul. Ecol. (Suppl. 3): 46-60.

NaKamura, K.; I. AbBAs \& A. Hasyim. 1988. Population dynamics of the phytophagous lady beetle, Epilachna vigintioctopunctata, in an egg plant field in Sumatra. Res. Popul. Ecol. 30: 25-41.

PARK, T. 1934. Observations on the general biology of the flour beetle, Tribolium confusum. Quart. Rev. Biol. 9: 36-54.

Phoofolo, M.W. \& J.J. ObRYCKI \& E.S. Krafsur. 1995. Temperature-Dependent Ovarian Development in Coccinella septempunctata (Coleoptera: Coccinellidae). Ann. Entomol. Soc. Amer. 88 (1): 72-79.

Precetti, A.A.C.M.; J. Milanez; J.R.P. PArRA \& E. Berti-Filho. 1977. Biologia e prejuizos causados por Epilachna cacica (Guérin, 1842) em aboboreira (Cucurbita moschata Duchesne). Ecossistema 2: 23-27.

Rajagopal, D. \& T.P. Trivedi. 1989. Status, bioecology and management of Epilachna vigintioctopunctata (Fab.) (Coleoptera: Coccinellidae) on potato in India: a review. Trop. Pest Manage. 35 (4): 410-413.

RiBeiro, C.S. \& L.M. AlmeidA. 1989. Descrição dos estágios imaturos de Epilachna spreta (Muls., 1850) (Coleoptera, Coccinellidae), com redescrição, comentários e chave para três outras espécies. Revta bras. Zool. 6 (1): 99-110.

Robertson, J.G. 1961. Ovariole Numbers in Coleoptera. Can. Jour. Zool. 39: 245-263.

SCHNEIDER, B.A. 1941. The nutritional requirements of Tribolium confusum Duval. I. The survival of adult beetles on patent flour and complete starvation diets. Biol. Bull. 80: 208-227.

Silva, A.G.; C.R. Gonçalves; D.M. Galvão; A.J.L. Gonçalves; J. Gomes; M.N. Silva; L. Simoni. 1968. Quarto catálogo dos insetos que vivem nas plantas do Brasil. Seus parasitos e predadores. Vol. 1, parte 2, Insetos, hospedeiros e inimigos naturais. Rio de Janeiro, Ed. Lab. Central de Patologia Vegetal, Ministério da Agricultura, 622p.

Simmons, L.W. 1988. The contribution of multiple mating and spermatophore consuption to the life time reproductive succes of male field crickets (Gryllus bimaculatus). Ecol. Entomol. 13: 57-69.

StewArt, L.A; A.F.G. Dixon; Z. Ruzick^ \& G. IPERTI. 1991. Clutch and egg size in ladybird beetles. Entomophaga 36 (3): 329-333.

WHEELER, D. 1996. The role of nourishment in oogenesis. Annu. Rev. Entomol. 41: 407-431.

WILSON, K.G.; R.E. STINNER \& R.L. RABB. 1982. Effects of temperature, relative humidity, and host plant on larval survival of the Mexican Bean beetle, Epilachna varivestis Muls.. Environ. Entomol. 11: $121-126$.

Recebido em 20.X.1999; aceito em 25.IV.2000. 